\title{
Shell-type SNRs as sources of cosmic rays
}

\author{
V.G. Sinitsyna ${ }^{a}$, M.S. Andreeva, K.A. Balygin, S.S. Borisov, I.A. Ivanov, A.M. Kirichenko, A.I. Klimov, \\ I.P. Kozhukhova, R.M. Mirzafatikhov, N.I. Moseiko, I.E. Ostashev, A.I. Palamarchuk, V.Y. Sinitsyna, and I.G. Volokh
}

P. N. Lebedev Physical Institute, Russian Academy of Science, Russia

\begin{abstract}
Investigations of VHE gamma-ray sources by any methods, including mirror Cherenkov telescopes, touch on the problem of the cosmic ray origin and, accordingly, the role of the Galaxy in their generation. SHALON observations have yielded results on Galactic supernova remnants (SNR) of different ages. Among them are: the shell-type SNRs Tycho's SNR (1572y), Cas A (1680y), IC 443 (age $\left.\sim(3 \div 30) \times 10^{3} \mathrm{y}\right)$, Cygni SNR (age $\left.\sim(5 \div 7) \times 10^{3} \mathrm{y}\right), \mathrm{G} 166.0+4.3\left(\right.$ age $\left.\sim 24 \times 10^{3} \mathrm{y}\right)$ and the classical nova GK Per (Nova 1901). Observation results are presented for each of the SNRs with spectral energy distributions by SHALON in comparison with other experiment data and images by SHALON together with data from X-rays by Chandra and radio-data by CGPS. The collected experimental data have confirmed the prediction of the theory about the hadronic generation mechanism of very high energy $800 \mathrm{GeV}-100 \mathrm{TeV}$ gamma-rays in Tycho's SNR, Cas A and IC443. For the first time, unique data on GK Per (Nova1901) TeV gamma-ray emission were obtained with the SHALON experiment. The X-ray data shows that the nova remnant of GK Per could be a younger remnant that will resemble older SNRs like IC 443 which interact with molecular clouds. GK Per is supposed to be a candidate for $\mathrm{TeV}$ gamma-ray emission due to accelerated particles in the reverse shock region.
\end{abstract}

\section{Introduction}

SHALON observations have yielded the results of two types of Galactic supernova remnants (SNR) of different ages. Among them are the shell-type SNRs: Tycho's SNR, Cas A, IC 443, $\gamma$ Cygni SNR, G166.0 + 4.3 as well as the classical nova GK Per that is in the earliest SNR evolutionary stage. For each of the SNRs the observation results are presented in Table 1 and Fig. 1 with the spectral energy distribution by SHALON ( $\triangle$ and $\boldsymbol{\Delta}$ ) compared with other experimental data and images by SHALON (color scale) in comparison with data from X-ray and radio-data. Also the theoretical predictions are shown. The collected experimental data can help to solve the cosmic ray origin.

\section{GK Per (Nova 1901)}

Nova Persei 1901 (GK Per) is one of the most extensively studied classical nova shells over the entire electromagnetic spectrum. The nova remnant is detected at radio energies with the Very Large Array as a source of nonthermal, polarized radio emission [1]. These observations show the existence of a shocked interstellar material. The X-ray shell around GK Per was first discovered with the ROSAT experiment and later was observed by the Chandra telescope [2]. The X-ray emission of the same electron population has been detected with Chandra as an extension from radio wavelengths. The detection of X-rays from the supernova remnant shell which are primarily due to bremsstrahlung of shock accelerated relativistic electrons, supposed the detection

a e-mail: sinits@sci.lebedev.ru of $\gamma$-ray emission originated from $\pi^{\circ}$-decay, secondary pp-interactions [3] as well as a possible contribution emission produced via Inverse Compton scattering [3]. Chandra $\mathrm{X}$-ray data shows that the nova remnant of GK Per could be a younger remnant that will resemble older SNRs like IC $443\left((3 \div 30) \times 10^{3} \mathrm{y}\right.$. $)$ interacted with molecular clouds.

In accordance with a program on long-term studies of metagalactic $\gamma$-ray sources, fifteen-year-long observations of the central galaxy in the Perseus cluster, NGC 1275, are being carried out in the SHALON experiment $[5,6]$. During the observations of NGC 1275 the SHALON field of view contains the source of nonthermal radio and X-ray emission GK Per (Nova 1901) of classical nova type as it is located $\sim 3^{\circ}$ North from NGC 1275. So due to the large telescopic field of view $>8^{\circ}$ the observations of NGC 1275 are naturally followed by the tracing of GK Per.

GK Per as a source accompanying NGC 1275 was observed with the SHALON telescope in the period from 1996 to 2012 for a total of 111 hours during clear moonless nights at zenith angles from $5^{\circ}$ to $35^{\circ}$. The observations were performed using the standard (for SHALON) technique of obtaining information about the cosmic-ray background and gamma-ray-initiated showers in the same observing session. The SHALON method of selecting $\gamma$-ray showers from background cosmic-ray showers allows a $99.92 \%$ rejection of the background showers [6]. The gamma-ray source associated with GK Per was detected above $2 \mathrm{TeV}$ by SHALON with a statistical significance $9.2 \sigma$ determined by the Li\&Ma method [4]. The signal significance for this SNR is less then one for the source with a similar flux and spectrum 
Table 1. The catalogue of galactic $\gamma$-ray sources observed by SHALON with parameters for spectrum fitting in the form of a power low with exponential cutoff $F(>E) \propto E^{k_{\gamma}} \times \exp \left(-E / E_{\text {cutoff }}\right)$.

\begin{tabular}{cccccc}
\hline Sources & Observable flux $^{a}$ & $k_{\gamma}$ & $E_{\text {cutoff }}, \mathrm{TeV}$ & Distance, kpc & Type \\
\hline Crab Nebula & $(2.12 \pm 0.12)$ & $-1.36 \pm 0.09$ & $19.0 \pm 2.0$ & 2.0 & PWN \\
Geminga & $(0.48 \pm 0.07)$ & $-0.39 \pm 0.05$ & $5.4 \pm 1.0$ & 0.25 & PSR or PWN \\
3C 58 & $(0.56 \pm 0.15)$ & $-1.33 \pm 0.12$ & - & 3.2 & PWN \\
G54.1+0.3 & $(0.97 \pm 0.35)$ & $-1.43 \pm 0.14$ & - & 6.2 & PWN \\
Tycho's SNR & $(0.52 \pm 0.04)$ & $-0.93 \pm 0.09$ & $35.0 \pm 5.0$ & $3.1-3.3$ & Shell-type SNR \\
Cas A & $(0.64 \pm 0.10)$ & $-0.91 \pm 0.11$ & $10.3 \pm 2.5$ & 3.1 & Shell-type SNR \\
IC 443 & $(1.63 \pm 0.23)$ & $-1.94 \pm 0.16$ & - & 1.5 & Shell-type SNR \\
rCygni SNR & $(1.27 \pm 0.11)$ & $-0.93 \pm 0.09$ & $20.1 \pm 4.2$ & 1.5 & Shell-type SNR \\
G166.0+4.3 & $(1.49 \pm 0.25)$ & $-1.95 \pm 0.44$ & - & 4.5 & Shell-type SNR \\
GK Per & $(0.31 \pm 0.14)$ & $-1.90 \pm 0.36$ & - & 0.46 & Classical Nova \\
Cyg X-3 & $(0.68 \pm 0.04)$ & $-1.15 \pm 0.08$ & $75.0 \pm 10.2$ & 10.0 & HMX Binary \\
4U 2129+47 & $(0.19 \pm 0.06)$ & $-0.42 \pm 0.12$ & $10.0 \pm 3.0$ & 6.0 & LMX Binary \\
Her X-1 & $(0.45 \pm 0.18)$ & - & - & 6.6 & LMX Binary \\
M57 & $(0.30 \pm 0.17)$ & - & - & 0.7 & Planetary nebula \\
\hline
\end{tabular}

${ }^{a}$ Integral flux at energy $>800 \mathrm{GeV}$ in units of $10^{-12} \mathrm{~cm}^{-2} \mathrm{~s}^{-1}$.

index obtained in the same observation hours because of a smaller collection field of view relative to the standard procedure of the SHALON experiment $[5,6]$. Corrections for the effective field of view were made to calculate the source flux and energy spectrum. The average integral flux at energies above $2 \mathrm{TeV}$ for $\mathrm{GK}$ Per is $I_{G K \text { Per }}=(2,9 \pm 1,3) \times 10^{-13} \mathrm{~cm}^{-2} \mathrm{~s}^{-1}$ (Fig. 1). Taking into account the proximity to a nearby source NGC 1275, we made the observation data procession first associated with NGC 1275 and then with GK Per. We found that $1 \%$ of showers are common for both sources. After a detailed analysis of the arrival direction and angular distance of these showers [6], less than $0.5 \%$ of NGC 1275 showers were recognized to be GK Per showers. This didn't change the average flux of NGC 1275 form [6]. The energy spectrum of gamma-rays in the observed energy region from 2 to $15 \mathrm{TeV}$ is well described by the power law $F(E>2 \mathrm{TeV}) \propto E^{k_{\gamma}}$, with $k_{\gamma}=-1.90 \pm 0.35$. The spectral energy distribution, detailed images of GK Per are shown in Fig. 1 and [5]. Two TeV $\gamma$-ray emission regions were revealed: the main one coinciding with the position of the central source of GK Per and the weak emission of the shell, that is also observed in X-rays by Chandra [2] (Fig. 1, right, red lines).

\section{Cas $A$ supernova remnant (1680 year)}

Cas A is a youngest of historical supernova remnants in our Galaxy. The supernova explosion that gave rise to Cas A occurred around 1680. Cas A was observed by SHALON during a 74 hour period of 2010-2014 [7,8]. The $\gamma$ ray source associated with $\mathrm{Cas} \mathrm{A}$ was detected above $800 \mathrm{GeV}$ at a level of $18.1 \sigma$ (the $\gamma$-ray flux and spectrum details see [8] and Table 1). Figure 1, left presents the spectral energy distribution of the $\gamma$-ray emission from Cas A by SHALON $(\boldsymbol{\Delta})$ in comparison with theoretical predictions $[10,11]$ and other experimental data (see refs in $[7,8])$. Figure 1 right presents the Chandra X-ray image of Cas A (red lines) [9] in comparison with the $\mathrm{TeV}$ structure in the energy range of $0,8-30 \mathrm{TeV}$ seen by SHALON.

The $\gamma$-ray emission in Cas A could be produced via IC scattering and by accelerated cosmic ray hadrons through interaction with the interstellar gas and then $\pi^{\circ}$ - decay.
Solid lines in Fig. 1 show the very high energy $\gamma$-ray spectra of hadronic origin [10,11]. It was shown in [10] that the leptonic model with $\mathrm{B}=0.3 \mathrm{mG}$ predicts a 58 times lower $\gamma$-ray flux than observed; the model with $\mathrm{B}=0.12 \mathrm{mG}$, which can broadly explain the observed $\mathrm{GeV}$ flux predicts the TeV spectrum with a cut-off energy about $10 \mathrm{TeV}$. The detection of $\gamma$-ray emission at $5-30 \mathrm{TeV}$ by SHALON and the hard spectrum below $1 \mathrm{TeV}$ would favor the $\pi^{\circ}$-decay origin of the $\gamma$-rays in Cas A.

\section{Tycho's Supernova Remnant (1572 year)}

Tycho's SNR originated from the Ia type supernova which exploded in 1572. The supersonic expansion of the stellar debris visible in Tycho's SNR by Chandra has created two X-ray emitting shock waves: moving outward into the interstellar gas, and another moving back into the debris. The character of such a displacement of the shock and the contact discontinuity surfaces makes cosmic ray acceleration at the supernova shock very efficient.

In the observations of 1996 a new galactic source was detected by SHALON [12] in TeV energies with $17 \sigma$ determined by [4]. This object was identified with Tycho's SNR. Tycho's SNR was also confirmed with VERITAS in observations of 2008-2010. The $\gamma$-ray emission from Tycho's SNR was detected with Fermi LAT in the range $400 \mathrm{MeV}-100 \mathrm{GeV}$. The expected flux of $\gamma$-quanta from $\pi^{\circ}$-decay, extends up to $>30 \mathrm{TeV}$, while the flux of $\gamma$-rays originating from IC scattering has a sharp cutoff above a few $\mathrm{TeV}$, so the detection of $\gamma$-rays with energies up to $80 \mathrm{TeV}$ by SHALON [7] (Fig. 1) is an evidence of their hadronic origin. Also, the information on parameters of Tycho's SNR such as the distance (3.1-3.3 kpc) and interstellar medium density was obtained from the SHALON data within the nonlinear kinetic model [13]. The same parameters have been obtained in [14] calculations of structures visible by Chandra at $\mathrm{X}$-ray energies (see red contours in Fig. 1, right).

\section{5. $\gamma$ Cygni SNR (age $\sim(5 \div 7) \times 10^{3}$ years)}

$\gamma$ Cygni SNR is a shell-type supernova remnant, its shelllike features are known in radio- and X-ray energy 

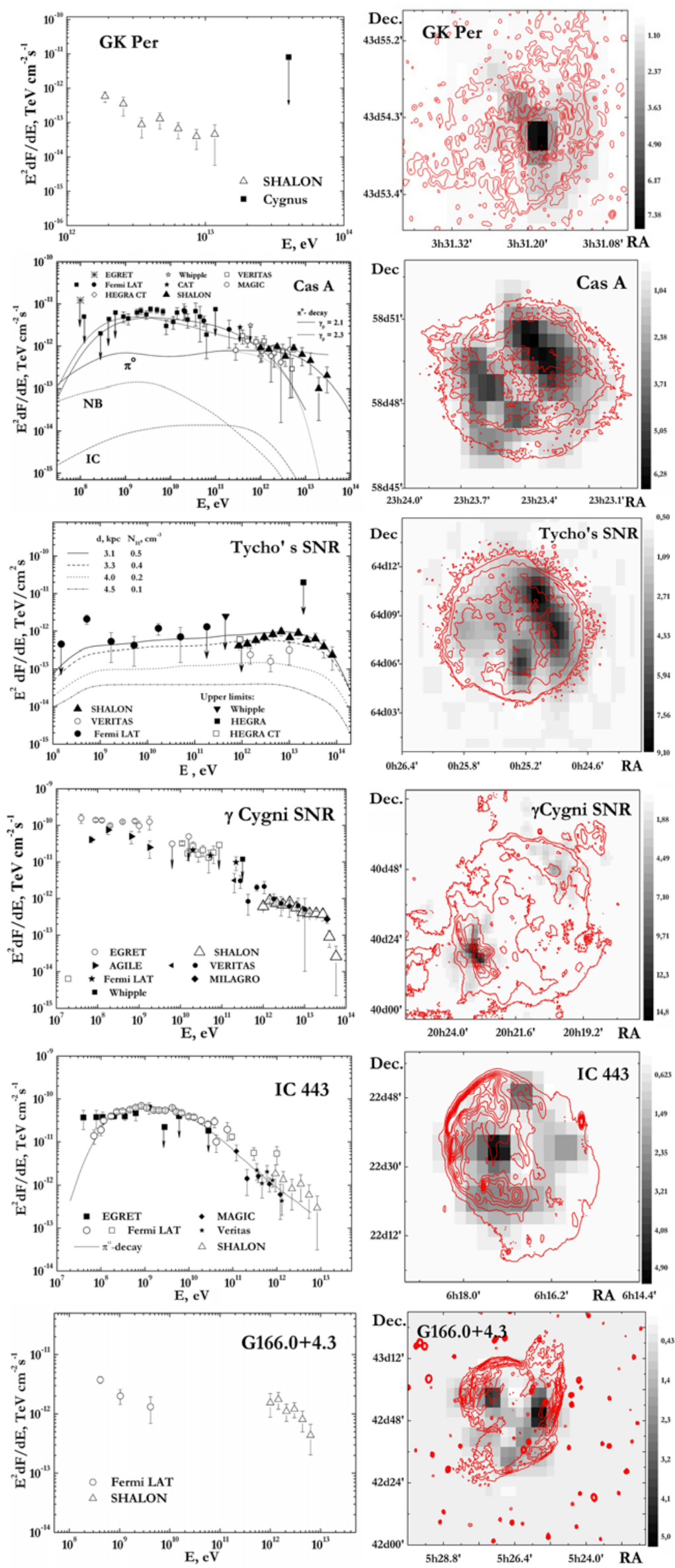

Figure 1. Spectral energy distributions (left) and images (right) of shell-type SNRs by SHALON compared with other experiments. 
regions [15]. $\gamma$ Cygni SNR is older than Cas A and Tycho's SNR, its age is estimated as 5000-7000 years [15] and is supposed to be in an early phase of adiabatic expansion.

During the observations of Cyg X-3 (since 1995) the SHALON field of view contains the $\gamma$ Cygni SNR as it is located in the Cygnus Region at $\sim 2^{\circ} \mathrm{SW}$ from Cyg $\mathrm{X}-3$. So due to the large telescopic field of view $\left(\sim 8^{\circ}\right)$ the observations of Cyg X-3 are naturally followed by observations of this SNR. As a result, $\gamma$ Cygni SNR was detected above $800 \mathrm{GeV}$ by SHALON (see $[8,16]$ ). TeV $\gamma$-ray emission regions correlate with the NW and SE parts of the shell visible in radio energies by CGPS (red contours in Fig. 1).

\section{IC 443 SNR (age $\sim(3 \div 30) x$ $10^{3}$ years)}

IC 443 is a shell-type SNR with a complex shape consisting of two half-shells with different radii. IC 443 is one of the best candidates for investigating the connection among SNRs, molecular clouds and high- and very high energy $\gamma$-ray sources. The close placement of the dense shocked molecular clouds and detected GeV-TeV $\gamma$-ray emission [18] suggests that IC 443 can be considered as a candidate for a hadronic cosmic-ray source.

IC 443 was detected by SHALON with an integral flux above $0.8 \mathrm{TeV}$ (see Table 1, [8,17] and Fig. 1) and a statistical significance of $9.7 \sigma$ [4]. The favored scenario in which the $\gamma$-rays of $100 \mathrm{MeV}-10 \mathrm{TeV}$ energies are emitted in the shell of the IC443 SNR is $\pi^{\circ}$-decay which are produced in the interactions of cosmic rays with the interstellar gas [18]. The solid lines in Fig. 1 show the $\gamma$-ray spectra of hadronic origin. Inverse Compton scattering can not explain the observed IC $443 \gamma$-ray emission as there is no bright source of seed photons in the region of IC 443. The analysis of arrival directions of $\gamma$-rays with energies $800 \mathrm{GeV}-10 \mathrm{TeV}$ reveal the correlation of $\mathrm{TeV}$ $\gamma$-ray emission maxima with $\mathrm{MeV}-\mathrm{GeV}$ emission observed by Fermi LAT [18]; also TeV $\gamma$-ray emission of South and South-West parts of IC 443 shell correlated with the position of the swept out dense molecular cloud (see red contours by CGPS in Fig. 1, right).

\section{7. $\mathrm{G} 166.0+4.3$ (age $\sim 24 \times 10^{3}$ years)}

$\mathrm{G} 166.0+4.3$ (VRO 42.05.01) is a supernova remnant with unusual morphology, containing a shell of circular component in the northeast intersected by a larger bowlshaped component in the southwest (the wing), expanding into a low density medium [19]. There are important differences in the spatial distribution of the emission viewed in $\mathrm{X}$-ray and radio energies. The radio image has an outstanding edge-brightened shape in the shell and wing component whereas the X-ray emission is not edge-brightened, but is dominated by a bright spot in the wing component [19]. As such remnant morphology can be results from the shock encountering a density discontinuity in the interstellar medium, G166.0 + 4.3 became a candidate for the investigation of particle acceleration in SNR shocks at high- and very high energies.

G166.0 + 4.3 was observed with the SHALON telescope during a period of 20.1 hours in 2015-2016.
The gamma-ray source associated with the G166.0 + 4.3 SNR was detected above $800 \mathrm{GeV}$ with a statistical significance of $6.1 \sigma$ with a gamma-quantum flux above $0.8 \mathrm{TeV}$ of $(1.49 \pm 0.25) \times 10^{-12} \mathrm{~cm}^{-2} \mathrm{~s}^{-1}$. The energy spectrum of gamma-rays in the observed energy region from $0.8 \mathrm{TeV}$ to $7 \mathrm{TeV}$ is well described by the power law $I\left(>E_{\gamma}\right)=(1.49 \pm 0.25) \times 10^{-12} \times E_{\gamma}^{-1.95 \pm 0.44}$. For each of the SNRs the observation results are presented in Table 1 and Fig. 1. The spectral energy distribution of G166.0 + 4.3 by SHALON together with Fermi LAT [20] data is presented in Fig. 1, left. Figure 1 right presents radio contours of $\mathrm{G} 166.0+4.3$ in comparison with the $\mathrm{TeV}$ structure in the energy range of $0,8-7 \mathrm{TeV}$ by SHALON. $\mathrm{TeV} \gamma$-ray emission regions correlate with the NE and SW parts of components visible in the radio energies by CGPS (red contours in Fig. 1, right). But TeV emission is more significant near the west, where the extended wing component and maximum of X-ray emission viewed by ROSAT [19] are located.

\section{Conclusion}

The observation results of Galactic shell-types supernova remnants at different evolution stages for GK Per (Nova 1901), Cas A, Tycho's SNR, $\gamma$ Cygni SNR, IC 443 and G166.0 + 4.3 by SHALON mirror Cherenkov telescope are presented. The $\mathrm{TeV} \gamma$-ray emission of classical nova GK Per, that could be a shell-type supernova remnant in an early evolution stage, was detected for the first time by SHALON as well as $\mathrm{TeV} \gamma$-rays from the GK Per shell. The experimental data have confirmed the prediction of the theory about the hadronic generation mechanism of very high energy $\gamma$-rays in Tycho's SNR, Cas A and IC443.

\section{References}

[1] S.P. Reynolds, R.A. Chevalier, Astrophys. J. 281, L33 (1984)

[2] S. Balman, Astrophys. J. 627, 933 (2005)

[3] E. G. Berezhko, H. J. Völk, Astropart. Phys. 7, 183 (1997)

[4] T.-P. Li and Y.-Q. Ma, Astrophys. J. 272, 317 (1983)

[5] V.G. Sinitsyna and V.Yu. Sinitsyna, Bull. of the Lebedev Phys. Inst. 42(6), 169 (2015)

[6] V.G. Sinitsyna and V.Yu. Sinitsyna, Astron. Lett. 40(2-3), 75 (2014)

[7] V.G. Sinitsyna and V.Yu. Sinitsyna, Astron. Lett. 37(9), 621 (2011)

[8] V.G. Sinitsyna and V.Yu. Sinitsyna "Astroparticle, Particle, Space Physics and Detectors Physics Applications" (Proc. 14th ICATPP, 2013) ed. S. Giani, C. Leroy, P.-G. Rancoita, L. Price and R. Ruchti (Singapore: World Scientific 2014), vol. 8, p. 135

[9] F.D. Seward, W.H. Tucker, R.A. Fesen, Astrophys. J. 652, 1277 (2006)

[10] A. A. Abdo et al., Astrophys. J. 710, L92 (2010)

[11] E.G. Berezhko, G. Pühlhofer, H.J. Völk, Astron. \& Astrophys 400, 971 (2003)

[12] V.G. Sinitsyna, et al. Nuclear Physics B (Proc.Suppl.) 97, 215 (2001)

[13] E.G. Berezhko, H.J. Völk, L.T. Ksenofontov, Astron. \& Astrophys. 483, 529 (2008) 
[14] D. Kosenko, S.I. Blinnikov, J. Vink, Astron. \& Astrophys. 532, 114 (2011)

[15] T.A. Lozinskaya, V.V. Pravdikova, A.V. Finogenov, Astron. Lett. 26, 77 (2000)

[16] V.G. Sinitsyna and V.Yu. Sinitsyna, Bull. of the Lebedev Phys. Inst. 40(5), 113 (2013)
[17] V.G. Sinitsyna and V.Yu. Sinitsyna, J. Phys.: Conf. Ser. 409, 012110 (2013)

[18] A.A. Abdo, et al., Astrophys. J. 712, 459 (2010)

[19] D.N. Burrows, Zh. Guo, Astrophys. J. 421(1), L19 (1994)

[20] M. Araya, MNRAS 434, 2202 (2013) 